\title{
Correlated variation of chiasma frequency and synaptonemal complex length in Locusta migratoria
}

\author{
C. QUEVEDO†, A. L. DEL CERRO†, J. L. SANTOS + AND G. H. JONES $\div *$ \\ $\nmid$ Departamento de Genetica, Facultad de Ciencias Biologicas, Universidad Complutense de Madrid, Madrid 28040, \\ Spain and $\ddagger$ School of Biological Sciences, The University of Birmingham, Edgbaston, Birmingham, B15 2TT, U.K.
}

\begin{abstract}
Mean diplotene chiasma frequency is shown to be significantly and positively correlated with mean synaptonemal complex (SC) length in 21 males of Locusta migratoria belonging to three different families. This may reflect a direct causal connection between SC length and chiasma frequency or an indirect relationship arising from the action of other factors with effects on both parameters. Various interpretations of the correlation are discussed in the light of old and new information on synaptic and recombinational processes during meiotic prophase I.
\end{abstract}

Keywords: chiasmata, Locusta migratoria, Orthoptera, recombination, synaptonemal complex.

\section{Introduction}

Despite recent doubts and questions concerning synaptonemal complex (SC) function(s) (Loidl, 1994; Kleckner, 1996), the impressive correlations of SC presence or SC length with chiasmata/crossovers (Jones, 1984; Loidl, 1994) ensure that a role for the SC in chiasma/crossover regulation will continue to be debated (Egel, 1995; Hasenkampf, 1996). Both SC length at pachytene and chiasma frequency at diplotene-metaphase I are known to show considerable variation from cell to cell within the same individuals. Since it is clearly impractical to measure these parameters at pachytene and then in the same cells at diplotene-metaphase $\mathrm{I}$, in order to investigate their relationship it is necessary to resort to estimating their mean values in populations of cells at pachytene and at diplotene-metaphase I, drawn from the same individuals.

There are in fact several indications in the literature that mean SC length at pachytene and mean chiasma frequency at diplotene-metaphase I (or mean crossover frequencies) show correlated variation. Most of these indications emerge from analyses of sex-related (male vs. female) differences in SC length and chiasma or crossover frequencies (Bojko, 1985; Wallace \& Hulten, 1985; Jones \& Croft, 1989 ), or from analysing the effects of additional

*Correspondence. E-mail: g.h.jones@bham.ac.uk

시 1997 The Genetical Society of Great Britain. heterochromatin in meiocytes, such as B chromosomes (Jones et al., 1989) or heterochromatic knobs (Mogensen, 1977). In all these cases, whatever the cause, higher chiasma/crossover frequencies are associated with longer SCs. An indication of the same relationship was found in wild population plants of Crepis capillaris, which was not dependent on sex difference or the presence of additional heterochromatin (de Azkue \& Jones, 1993), but this conclusion was very tentative because of the low number of plants included in the study. It therefore remains a priority to determine whether this relationship does obtain in 'normal' individuals, independently of any special effects such as sex difference or possession of heterochromatin. This study was initiated and designed specifically to address this question. Locusta migratoria was chosen as the experimental organism because of its ease of laboratory rearing, the large number of testicular follicles in males, the precision of chiasma scoring at diplotene (Henderson, 1970; Tease \& Jones, 1978) and its suitability for SC spreading (Croft \& Jones, 1986).

\section{Materials and methods}

The individual males of Locusta migratoria used in this study were derived from a laboratory stock by a mixture of random mating and sib-mating over four generations. The original stock was designated $F_{0}$. Three males from this stock were sib-mated to origi- 
nate the three families and the analysed individuals were $\mathrm{F}_{4}$. Random mating was imposed at the $\mathrm{F}_{0}$ and $F_{2}$ generations, and sib-mating at the $F_{1}$ and $F_{3}$ generations. Three $F_{4}$ families were analysed, each of which comprised full-sibs from an $F_{3}$ single-pair brother-sister mating. Seven individual males per family were analysed giving a total of 21 males.

Males of $L$. migratoria were reared in wooden cages, $40 \times 40 \times 40 \mathrm{~cm}$. They were kept in a controlled environment room maintained at $24^{\circ} \pm 2^{\circ} \mathrm{C}$ and 40 per cent relative humidity. A 40 watt bulb in each cage was used to establish a temperature of about $30^{\circ} \mathrm{C}$ during a 13 -hour day. They were fed on barley plantlets. Last instar nymphs were dissected and the testes of each individual were divided into two parts. One part was fixed in ethanol-glacial acetic acid (3:1) and stored at $4^{\circ} \mathrm{C}$, for chiasma analysis. The other part was used, without prior fixation, for SC spreading.

Chiasmata were analysed from squash preparations of fixed testes in 45 per cent acetic acid using phase contrast microscopy. Ten cells at the diplotene stage (Fig. 1) were analysed from each of three follicles per male, giving a total of 30 diplotene cells per male. No significant interfollicle variation in chiasma frequency was detected.

For SC spreading, testis follicles were carefully dissected to remove fat body and then homogenized

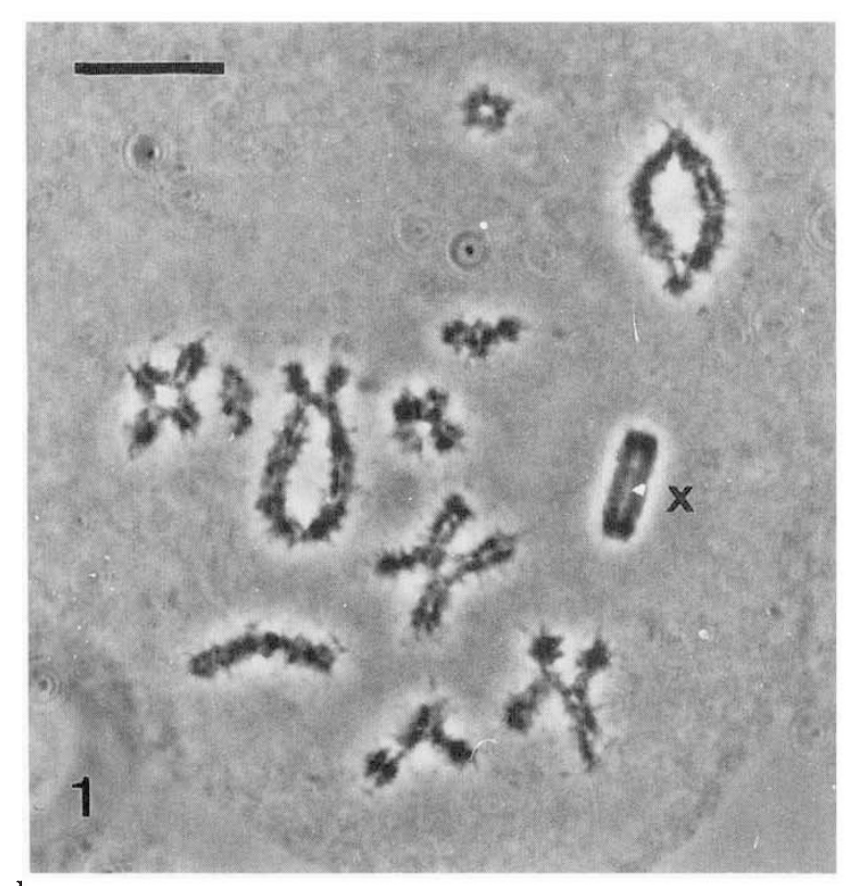

]

phase contrast microscopy, showing 13 chiasmata. $X=X$ chromosome. Scale bar $=15 \mu \mathrm{m}$. in Dulbecco's medium (Gibco; $4500 \mathrm{mg}$ D-glucose $\mathrm{L}^{-1}$ with L-glutamine and without $\mathrm{Na}$ pyruvate) containing $2 \mathrm{~mm}$ EDTA and 0.1 per cent bovine serum albumin. Two drops of spreading medium $\left(60 \mathrm{~mm} \mathrm{NaP0} 0_{4}\right.$ buffer, $1 \mathrm{~mm}$ EDTA, 0.03 per cent Triton $\mathrm{X}-100, \mathrm{pH} 7.5$ ) were added to a single drop of homogenized testis suspension on a cavity slide, left for 5-10 min and then washed onto slides with seven drops of paraformaldehyde fixative $(\mathrm{pH} \mathrm{8.9)}$. The preparations were then dried overnight on a warm plate at $30-35^{\circ} \mathrm{C}$, rinsed, and air dried. For silver impregnation, a few drops of 33 per cent $\mathrm{AgNO}_{3}$ solution were placed on the preparation, which was then covered with a patch of nylon gauze (Loidl \& Jones, 1986) and incubated in a moisture chamber at $60^{\circ} \mathrm{C}$ for $30 \mathrm{~min}$. Thirty well-spread pachytenes (Fig. 2) were examined from each male, using a Zeiss Ultraphot photomicroscope and photographed on Kodak Technical-Pan film. The absolute lengths of synaptonemal complexes were measured from enlarged photographic prints using a map measurer.

\section{Results and discussion}

Mean diplotene chiasma frequencies and mean pachytene SC lengths of 21 individual males of $L$. migratoria from three different families are summa-

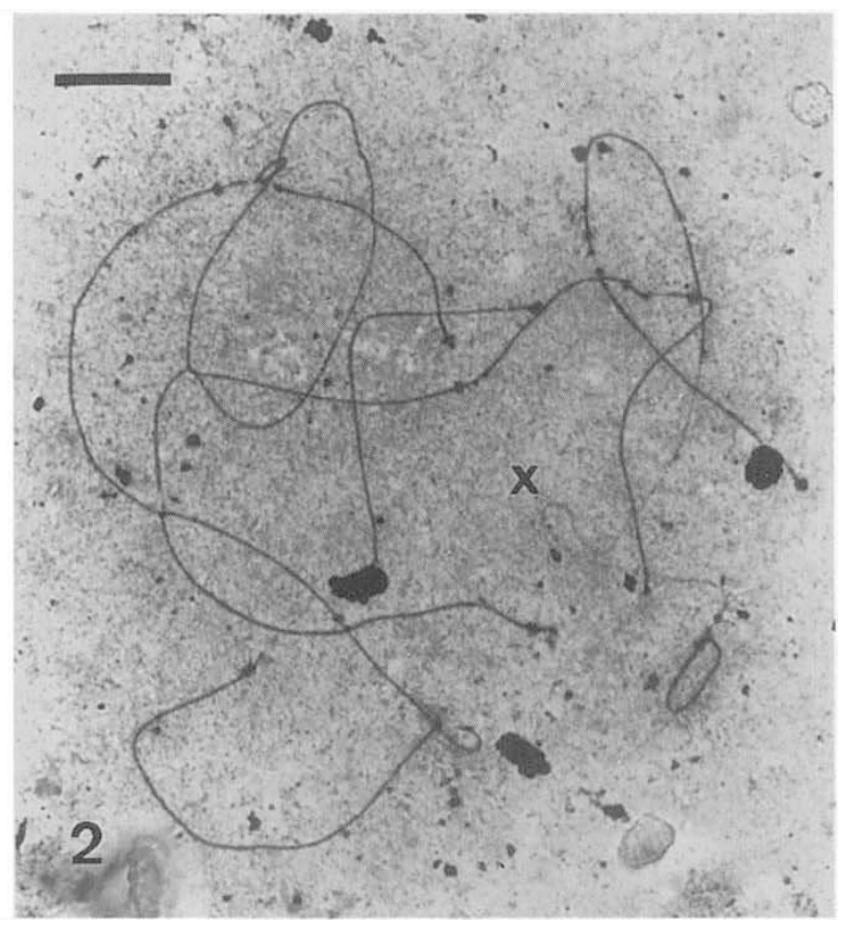

Fig. 2 Light micrograph of a silver-stained pachytene SC surface-spread. $X=X$ chromosome. Scale bar $=15 \mu \mathrm{m}$.

(C) The Genetical Society of Great Britain, Heredity, 78, 515-519. 
Table 1 A summary of SC length data and mean chiasma frequencies of 21 males of Locusta migratoria

\begin{tabular}{llcccccc}
\hline Family & Male & $\begin{array}{c}\text { Mean SC } \\
\text { length }(\mu \mathrm{m})\end{array}$ & SD & Range & $\begin{array}{c}\text { Mean chiasma } \\
\text { frequency }\end{array}$ & SD & Range \\
\hline B & B3 & 399.25 & 54.55 & $267.2-511.2$ & 13.60 & 0.62 & $13-15$ \\
B & B17 & 390.91 & 48.40 & $308.0-484.8$ & 14.17 & 0.70 & $13-15$ \\
B & B16 & 430.16 & 35.40 & $363.2-520.0$ & 14.63 & 1.07 & $13-17$ \\
B & B21 & 450.30 & 53.38 & $322.4-534.4$ & 15.20 & 0.89 & $14-17$ \\
B & B4 & 419.84 & 42.24 & $351.2-516.0$ & 15.53 & 0.94 & $14-18$ \\
B & B24 & 483.07 & 47.47 & $376.8-575.2$ & 15.83 & 0.95 & $14-18$ \\
B & B15 & 445.81 & 43.03 & $374.4-557.6$ & 16.13 & 0.86 & $14-17$ \\
C & C3 & 329.15 & 36.07 & $274.4-411.2$ & 12.83 & 0.79 & $11-14$ \\
C & C8 & 333.87 & 35.13 & $275.2-421.6$ & 13.70 & 0.91 & $12-16$ \\
C & C4 & 373.44 & 40.37 & $278.4-455.2$ & 14.00 & 1.02 & $12-16$ \\
C & C14 & 405.92 & 28.64 & $350.4-442.4$ & 14.40 & 0.67 & $13-15$ \\
C & C1 & 392.00 & 51.98 & $286.4-496.8$ & 14.80 & 0.85 & $13-16$ \\
C & C2 & 380.91 & 37.74 & $333.6-474.4$ & 15.07 & 1.50 & $13-17$ \\
C & C13 & 446.03 & 64.61 & $344.0-573.6$ & 15.40 & 0.85 & $14-17$ \\
D & D1 & 357.36 & 33.55 & $288.0-436.8$ & 13.10 & 0.80 & $12-14$ \\
D & D3 & 383.07 & 34.16 & $328.0-472.8$ & 13.93 & 0.69 & $13-15$ \\
D & D4 & 403.89 & 54.67 & $318.4-526.4$ & 14.33 & 0.92 & $13-16$ \\
D & D7 & 507.52 & 77.05 & $338.4-637.6$ & 15.13 & 1.01 & $13-17$ \\
D & D5 & 438.37 & 49.20 & $368.8-583.2$ & 15.30 & 0.79 & $14-17$ \\
D & D8 & 428.27 & 42.24 & $365.6-517.6$ & 15.67 & 0.84 & $14-17$ \\
D & D9 & 534.99 & 51.17 & $445.6-644.0$ & 16.27 & 0.91 & $15-18$ \\
\hline
\end{tabular}

rized in Table 1. Mean chiasma frequencies range from 12.83 per cell to 16.27 per cell in different individuals. The three families each show similar ranges of mean chiasma frequencies of about three chiasmata per cell, and the families do not differ significantly for this parameter $\left(F_{2,18}<1\right)$. Similarly, the three families show comparable ranges (25-50 per cent) of mean SC lengths and again do not differ significantly for this parameter $\left(F_{2,18}=2.962\right.$, $P>0.05$ ).

The relationship of mean chiasma frequencies and mean SC lengths of the 21 individual males is shown graphically as a scatter diagram (Fig. 3). Correlation analysis, rather than regression analysis is judged to be appropriate to this situation because there is no sound basis for judging either to be a dependent or independent variable. Initially the three families were analysed separately, yielding positive and statistically significant correlation coefficients in each case (Table 2). The three correlation coefficients were tested for homogeneity using the procedure given by Steel \& Torrie (1960, p.191). This test clearly establishes that the correlation coefficients are homogeneous $\left(\chi_{2}^{2}=0.178, P>0.9\right)$ and can be combined to give a pooled correlation coefficient which is, as expected, positive and highly significant $\left(r=0.840 \pm 0.124 ; t_{19}=6.742, P<0.01\right)$.

(c) The Genetical Society of Great Britain, Heredity, 78, 515-519.

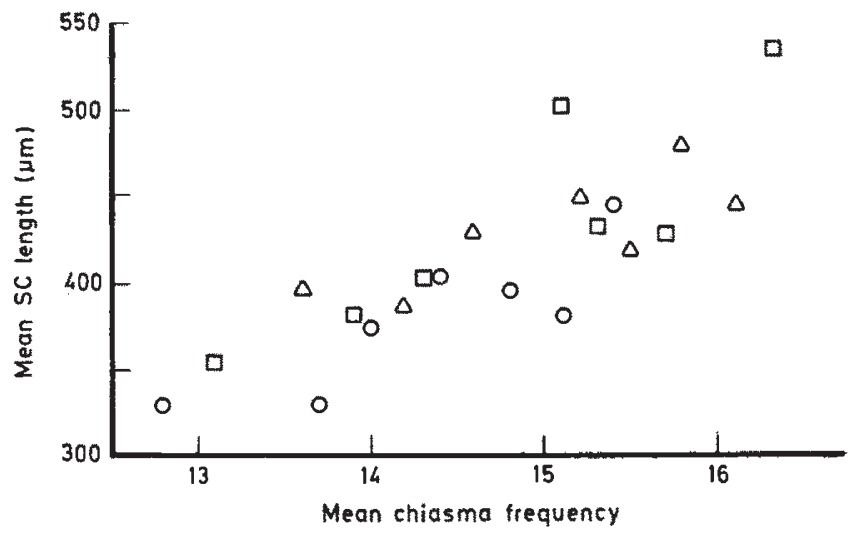

Fig. 3 Mean chiasma frequency plotted against mean SC length for 21 males of Locusta migratoria, belonging to three families designated $B(\triangle), C(O)$ and $D(\square)$.

This strong correlation of mean chiasma frequency and mean SC length in Locusta is impressive, but its meaning is far from clear. A parallel exists between this study and an earlier analysis by Fox (1973) in a different locust species, Schistocerca gregaria. He showed that chiasma frequency and bivalent length, both measured at diplotene, were correlated and furthermore he argued that the mechanism responsible for the correlation operated at a cellular level rather than at the level of indivi- 
Table 2 A summary of correlation coefficient $(r)$ analyses of mean chiasma frequency and mean SC length for 21 males of Locusta migratoria

\begin{tabular}{|c|c|c|c|c|c|c|c|c|}
\hline & \multicolumn{4}{|c|}{ Mean chiasma frequency vs. mean SC length } & \multicolumn{4}{|c|}{ Mean chiasma frequency vs. three shortest SCs } \\
\hline & $r$ & d.f. & $t$ & $P$ & $r$ & d.f. & $t$ & $P$ \\
\hline Family B & $0.847 \pm 0.238$ & 5 & 3.562 & $<0.02$ & $0.791 \pm 0.274$ & 5 & 2.892 & $<0.05$ \\
\hline Family $\mathrm{C}$ & $0.776 \pm 0.282$ & 5 & 2.751 & $<0.05$ & $0.603 \pm 0.357$ & 5 & 2.856 & $<0.05$ \\
\hline Family D & $0.867 \pm 0.223$ & 5 & 3.891 & $<0.02$ & $0.839 \pm 0.243$ & 5 & 3.448 & $<0.02$ \\
\hline All males & $0.840 \pm 0.124$ & 19 & 6.742 & $<0.001$ & $0.760 \pm 0.149$ & 19 & 4.586 & $<0.001$ \\
\hline
\end{tabular}

dual bivalents. Although conceding that it was not possible to distinguish cause and effect in this relationship, Fox nevertheless reflected the prevailing view of the time, that it seemed inherently more likely that chromosome length determines chiasma frequency, and not vice versa.

A similar cell-based control of the chiasma frequency/SC length relationship is evident in the present study. The three shortest bivalents in the Locusta karyotype, designated $\mathrm{M}_{9}, \mathrm{~S}_{10}$ and $\mathrm{S}_{11}$, almost invariably possess a single chiasma at diplotene; exceptionally a second chiasma may occur but so infrequently as to be discountable. Nevertheless, these three short bivalents show SC length variation which parallels that in the other, longer chromosomes, and this is strongly correlated with mean cell chiasma frequency, but not of course with the chiasma frequencies of the individual bivalents concerned.

These correlations are open to a number of interpretations. In the first place they may indicate a direct causal connection between the parameters under consideration, which could of course operate in either direction. Because chiasmata per se, as physical entities, materialize only after the dissolution of the SC, the only possible direct causal connection is that $\mathrm{SC}$ length influences chiasma frequency. As several commentators have suggested a role for the SC in regulating chiasmata (King \& Mortimer, 1990; Egel, 1995; Hasenkampf, 1996), the proposal that SC length may influence chiasma frequency is entirely plausible and is consistent with many other observations and arguments. For instance, if interference is transmitted via the SC, and average interference distances are relatively constant for particular bivalents then longer SCs should result in more chiasmata.

However, we must also consider the possibility that chiasma frequency and SC length are both influenced by one or more other factors, in which case the correlations are merely statistical effects and do not imply direct causality. This underlying factor might be some aspect or aspects of reciprocal recombination events, which will of course eventually mature into chiasmata and may also have effects on SC length. Because it is now recognized that, at least in yeast, the initial events of recombination occur very early in meiotic prophase, preceding SC formation, and the maturation of recombination intermediates into full reciprocal recombinants occurs during pachytene (Padmore et al., 1991), this proposition is entirely feasible. Alternatively, the condition of chromosome axes and their associated chromatin during early prophase I could be key factors in determining reciprocal recombination (and hence chiasma) frequencies and would also be likely to influence SC length at pachytene. In this context it is interesting to note that organisms with low DNA/SC ratios generally have high recombination frequencies (Loidl, 1994). Indeed there is a correlation between the less dense DNA packaging and increased rate of recombination displayed by human-derived YACs compared with human DNA in its natural environment (Loidl et al., 1995).

Given our present state of knowledge, it is not possible to discriminate between the various interpretations discussed above. It remains clear, however, that mean SC lengths and mean chiasma frequencies of individual males of $L$. migratoria show a striking positive correlation, and this, together with the parallel observations from other species, requires to be explained by any comprehensive hypothesis of the genesis, maturation and regulation of meiotic reciprocal recombination events.

\section{Acknowledgement}

This work was partly supported by the European Economic Community (contract CHRX-CT940511) and by grant PB95-0421 awarded by the Direccion General de Investigacion Cientifica $y$ Tecnica (DGICYT, Spain). 


\section{References}

војко, м. 1985. Human meiosis IX. Crossing over and chiasma formation in oocytes. Carlsberg Res. Comm., 50, 43-72.

CROFT, J. A.. AND JONES, G. H. 1986. Surface spreading of synaptonemal complexes in locusts. I. Pachytene observations. Chromosoma, 93, 483-488.

DE AZKUE, D. AND JONES, G. H. 1993. Inter- and intra-plant SC length variation in Crepis capillaris. Heredity, 71, 363-368.

EGEL, R. 1995. The synaptonemal complex and the distribution of meiotic recombination events. Trends Genet., 11, 206-208.

Fox, D. P. 1973. The control of chiasma distribution in the locust, Schistocerca gregaria (Forskal). Chromosoma, 43, 289-328.

HASENKAMPF, C. A. 1996. The synaptonemal complex-the chaperone of crossing over. Chromosome Res., 4, $133-140$.

HENDERSON, S. A. 1970. The time and place of meiotic crossing-over. Ann. Rev. Genet., 4, 295-324.

JONES, G. H. 1984. The control of chiasma distribution. In: Evans, C. W. and Dickinson, H. G. (eds) Controlling Events in Meiosis. Symp. Soc. Exp. Biol., 38, 291-320.

JONES, G. H. AND CROFT, J. A. 1989. Chromosome pairing and chiasma formation in spermatocytes and oocytes of Dendrocoelum lacteum (Turbellaria: Tricladida); a cytogenetical and ultrastructural study. Heredity, 63, 97-106.

JONES, G. H., WHITEHORN, J. A. F. AND ALBINI, S. M. 1989. Ultrastructure of meiotic pairing in B chromosomes of Crepis capillaris. I. One-B and two-B pollen mother cells. Genome, 32, 611-621.

KING, J. S. AND MORTIMER, R. K. 1990. A polymerization model of chiasma interference and corresponding computer simulation. Genetics, 126, 1127-1138.

KLeCKner, N. 1996. Meiosis: how could it work? Proc. Natl. Acad. Sci. U.S.A., 93, 8167-8174.

LoIDL, J. 1994. Cytological aspects of meiotic recombination. Experientia, 50, 285-294.

LOIDL, J. AND JONES, G. H. 1986. Synaptonemal complex spreading in Allium. I. Triploid A. sphaerocephalon. Chromosoma, 93, 420-428.

LOIDL, J., SCHERTHAN, H., DEN DUNNEN, J. T. AND KLEIN, F. 1995. Morphology of a human-derived YAC in yeast meiosis. Chromosoma, 104, 183-188.

MOGENSEN, H. L. 1977. Ultrastructural analysis of female pachynema and the relationship between synaptonemal complex length and crossing-over in Zea mays. Carlsberg Res. Comm., 42, 475-497.

PADMORE, R., CAO, L. AND KLECKNER, N. 1991. Temporal comparison of recombination and synaptonemal complex formation during meiosis in S. cerevisiae. Cell, 66, $1239-1256$.

STEEL, R. G. D. AND TORRIE, J. H. 1960. Principles and Procedures of Statistics. McGraw-Hill, New York.

TEASE, C. AND JONES, G. H. 1978. Analysis of exchanges in differentially stained meiotic chromosomes of Locusta migratoria after BrdU-substitution and FPG staining. I. Crossover exchanges in monochiasmate bivalents. Chromosoma, 69, 163-178.

WALLACE, B. M. N. AND HULTEN, M. 1985. Meiotic chromosome pairing in the normal human female. Ann. Hum. Genet., 49, 215-226. 